\title{
Boundary shapes guide selection of reference points in goal localization
}

\author{
Ruojing Zhou ${ }^{1} \cdot$ Weimin Mou ${ }^{2}$ \\ Published online: 26 June 2019 \\ (C) The Psychonomic Society, Inc. 2019
}

\begin{abstract}
In this study, we contrasted two hypotheses theorizing the role of the global shape of a boundary in object location memory: People might differentiate reference points based on the global shape extracted from the environment configuration and choose appropriate parts for encoding a specific location, or, alternatively, only the number of reference points provided by a shape might be important for accurate encoding. We designed a location memory task in an immersive virtual environment in order to examine these two hypotheses. Participants first learned four target locations with a circular wall and a landmark array. During testing, participants recalled the locations with either one entire cue or part of one cue removed. Location memory was impaired when the testing cues did not form a circle, but it was not impaired when the testing configuration retained the circular shape. In Experiment 2, the circle formed by a landmark array and the circular wall did not share the same center during learning. Memory performance decreased when either the wall or the landmark array was removed during testing. These results indicated that participants might segment the shape of the circular wall into parts (similar to segmenting a clock face into 12 hours) and encode target locations relative to the differentiated parts. When such segmentation could be recovered from the testing configuration, object location memory was retained. Otherwise, impairment occurred during testing. These findings suggest that although the individual reference points on a boundary are important for encoding specific target locations, the global shape of the boundary nonetheless affects segmentation and the selection of individual reference points.
\end{abstract}

Keywords Object location memory $\cdot$ Boundary $\cdot$ Landmark $\cdot$ Cue competition $\cdot$ Shape segmentation

Finding a target location is a common and important task that humans and most nonhuman animals practice daily. Animals must remember where their food has been cached for later retrieval. Human field workers who work outdoors must remember the locations of their home base and working area. The environment in which humans and nonhuman animals navigate usually contains rich cues that can be used to encode locations in memory, and such representations can guide goal localization behavior (e.g., finding a target). Two types of

Ruojing Zhou

Ruojing.Zhou@dzne.de

Weimin Mou wmou@ualberta.ca

1 German Center for Neurodegenerative Diseases (DZNE), Magdeburg, Germany

2 University of Alberta, Edmonton, Alberta, Canada environmental cues have been identified to serve as reference points for goal localization (Doeller \& Burgess, 2008; Doeller, King, \& Burgess, 2008; for a review, see Lew, 2011): a distinctive local landmark (such as a building on campus) or a continuous boundary (such as a wall surrounding a campus).

It has been proposed and empirically demonstrated that encoding a target location relative to a single landmark involves a learning mechanism distinct from that underlying encoding locations relative to a boundary (Bird, Capponi, King, Doeller, \& Burgess, 2010; Bullens et al., 2010; Doeller \& Burgess, 2008; Doeller et al., 2008). In one experiment by Doeller and Burgess, in addition to distal orientation cues, participants remembered four objects' locations with the presence of both a circular boundary and a single landmark within the boundary (compound-learning-cue groups) or with the presence of either of these two cues (single-learning-cue groups). In the testing phase, the compound-learning-cue groups replaced the targets with the boundary alone (the landmark removed) or the landmark alone (the boundary 
removed). The single-learning-cue groups replaced the targets with the presence of the same cue that had been shown during learning. The results showed that when the landmark was presented as the testing cue, less accurate location memory was observed in the compound-learning-cue group than in the single-learning-cue group. In contrast, when the boundary was presented as the testing cue, comparably accurate location memory was observed in the compound- and single-learningcue groups. These results indicated that boundary-related encoding impaired (overshadowed) landmark-related encoding, but not vice versa. The authors concluded that encoding a location relative to a boundary follows the latent/ incidental-learning rule, whereas encoding a location relative to a single landmark follows the associative-learning rule. Doeller, King, and Burgess (2008) further proposed that what makes boundaries different from individual landmarks at the neural computation level is that the place cells in the hippocampal system are tuned to a boundary rather than to a single landmark.

In a follow-up study to Doeller and Burgess (2008), Mou and Zhou (2013) distinguished two factors that might contribute to the "boundaryness" of a continuous surface: the large number of reference points provided by a boundary, or the extended surface. Mou and Zhou hypothesized that the finding that boundary-related encoding overshadowed landmarkrelated encoding, but not vice versa, might be attributed to the large number of reference points in a boundary rather than to the extended surface. A continuous boundary contains an infinite number of reference points, which allows vectors from multiple directions to be established between the boundary and a target location. In contrast, a single landmark may provide only one reference point, and thus allows for only one vector between the landmark and a target location. In the experiment of Doeller and Burgess, for the compound-learningcue conditions, when the landmark was removed, only one vector was withdrawn, whereas when the boundary was removed, all vectors but one were withdrawn. Therefore, object location memory decreased in the latter but was maintained in the former case. Mou and Zhou referred to this hypothesis as the multiple-reference-point hypothesis.

Mou and Zhou (2013) provided empirical evidence supporting the multiple-reference-point hypothesis. As in Doeller and Burgess's (2008) paradigm, four conditions were employed in the first experiment of the study: two single-cue conditions, in which participants learned four objects' locations and were tested subsequently with the same cues, either a circular wall (the boundary condition, referred to as $B$ ) or a single traffic cone (the landmark condition, referred to as $L$ ); and two compound-cue conditions, in which participants learned with both the boundary and the landmark but were tested with either the circular wall (referred to as $B L-B$ ) or the traffic cone (referred to as $B L-L$ ). Object location memory was assessed as participants replaced the objects in their remembered locations. Distance errors were recorded, as measured by the distance between a response location and the corresponding correct location. The results showed that larger distance errors in the testing phase than in the learning phase occurred in the BL-L group, but not in the other three groups, including the BL-B group. These results indicated that the boundary-related encoding impaired the landmark-related encoding, whereas the landmark-related encoding did not impair the boundary-related encoding, consistent with the overshadowing effect in Doeller and Burgess's study. We refer to the larger distance errors in the testing than in the learning phase as the impairment effect.

Importantly, in a second experiment, Mou and Zhou (2013) replaced the single cone with an array of cones forming a circle that was concentric with the circular wall. Again, the two compound-cue condition (referred to as $B L-L$ and $B L-B$, where $L$ refers to the landmark array) and the two single-cue conditions (referred to as $L$ and $B$ ) were employed. Strikingly, there was no impairment effect in any of the four groups. In their last experiment, Mou and Zhou systematically manipulated the number of the cones (from two to 24). The results showed that the magnitude of the impairment effect in the $\mathrm{BL}-\mathrm{L}$ group was negatively correlated with the number of the cones when the array consisted of 12 or fewer cones; the impairment effect was, however, eliminated when there were 18 or more traffic cones in the array. Therefore, an array of evenly spaced cones (e.g., 18 or more cones) could function as a boundary, in the sense of no impairment effect upon the removal of the continuous wall at test. Since the array of cones did not have any continuous surface, the large number of reference points rather than the extended surface of a boundary might have caused the findings that boundary-related encoding impaired (overshadowed) landmark-related encoding, but not vice versa, supporting the multiplereference-point hypothesis.

The multiple-reference-point hypothesis can be regarded as an expansion of the boundary-vector-cell (BVC) model originally proposed by O'Keefe and Burgess (1996; see also Barry \& Burgess, 2014; Barry et al., 2006). According to the BVC model, a place cell receives its input from an ensemble of boundary-vector cells that are tuned to barriers (extended surfaces) from multiple directions with a fixed distance to a navigator; the firing rate of the place cell is the thresholded sum of the inputs from the boundary-vector cells (Barry \& Burgess, 2014; Barry et al., 2006). According to the multiple-referencepoint hypothesis, the accuracy of location memory increases with the number of reference points. Therefore, these two models are essentially similar, except that the multiplereference-point hypothesis stipulates that the vectors to the target can originate not only from extended surfaces, but also from discrete objects. The multiple-reference-point hypothesis is also similar to the vector-sum model proposed by Cheng $(1988,1989)$ to explain how pigeons use landmarks to find a 
location (see also the multiple-bearings hypothesis for nutcrackers' caching behavior; Kamil \& Cheng, 2001). According to the vector-sum model, pigeons record a number of "landmark-to-goal" vectors from the goal position, and these vectors contain both distance and directional information. Furthermore, these vectors are assigned with different weightings, with larger weightings to the closer landmarks, as reflected by the observation that shifting the positions of different landmarks exerted controls to different degrees on pigeons' searching locations for the same target (Cheng, 1989).

All three of the models discussed above give credit to the individual vectors established between multiple reference points and a target location. However, according to these models, the global shape formed by the multiple reference points might not be critical in the localization process. In particular, in Mou and Zhou (2013), participants might encode one target location relative to all the reference points available during learning. The accuracy of location memory during testing would not decrease, as long as enough reference points (e.g., 18 or more cones) were available at retrieval. When the number of the reference points at retrieval was not sufficient, the accuracy of object location memory decreased linearly with the decrease in the number of the cones presented during testing. We refer to this stipulation as the sufficient-referencepoints hypothesis, since it only regards the number of reference points at retrieval as the determinant for accuracy in location memory. Below we will propose an alternative account that also emphasizes the role of the global shape information derived from the configuration of multiple reference points.

Although the function of global shapes in goal localization has been less investigated, the function of global shapes in reorientation has been reported prevalently (see Cheng \& Newcombe, 2005, for a review). In his seminal work, Cheng (1986) trained rats to find food located at one of the four corners in a rectangular enclosure after disorientation. Despite the fact that the enclosure contained highly informative featural information (such as distinctive panels at each corner), rats seemed to rely on the global geometry of the enclosure (i.e., the rectangular shape) in order to reorient themselves: They searched mainly at the correct corner and at its opposite diagonal corner, which is geometrically identical to the correct corner, but failed to distinguished the two by the unique features available at the corners. Since this original observation, the reorientation paradigm has been widely employed to test various species in different environmental settings (Hermer \& Spelke, 1994; Kelly, Spetch, \& Heth, 1998; Sovrano, Bisazza, \& Vallortigara, 2002; Vallortigara, Zanforlin, \& Pasti, 1990; for a review, see Cheng, Huttenlocher, \& Newcombe, 2013). Although early reorientation research has started a debate on the universal dominance of geometric information in controlling the reorientation process across species (e.g., Cheng et al., 2013; Learmonth, Nadel, \& Newcombe, 2002; Lee \& Spelke, 2010; Lew, 2011), growing evidence from the last decade has suggested that boundary primacy is not always observed. Studies using rats (e.g., Pearce, Graham, Good, Jones, \& McGregor, 2006) as well as humans (e.g., Buckley, Smith, \& Haselgrove, 2019) have demonstrated that learning of the geometric shape imposed by a boundary can be interfered with by learning of nongeometric cues (e.g., overshadowing and blocking).

Extending the important role of global shapes in reorientation, we propose a shape-based segmentation model that acknowledges the importance of the global shape of a boundary in goal localization, especially in selecting the reference points from the boundary to which target locations are encoded. According to the model, people first segment a boundary on the basis of the shape of the boundary. If the shape of the boundary indicates a clear orientation - for example, an isosceles triangle - the reference points on the boundary can be differentiated on the basis of the shape itself. If the shape of the boundary does not indicate a clear orientation - for example, a circle - the reference points on the boundary are differentiated on the basis of either the distal orientation cues (e.g., Doeller \& Burgess, 2008) or inertial locomotion cues (e.g., Foo, Warren, Duchon, \& Tarr, 2005; Yoder, Clark, \& Taube, 2011). After this initial segmentation, people specify the location of a target relative to one or several differentiated local segments (i.e., segments that are closer to the target) by establishing vectors between the segments and the target. For instance, to specify the location of a target in a square room, one could divide the room into four corners and four walls. Using orientation information available in the room, which could originate from a distal landmark or from inertial locomotion, one could distinguish the four otherwise identical corners and the walls. One could then choose a differentiated corner or a wall as a reference point for encoding the target location. We refer to this model as the shape-segment hypothesis. The shape-segment process is in line with the ideas of differentiating locally similar corners based on a global shape (Miller \& Shettleworth, 2007).

We contrasted the shape-segment hypothesis with the sufficient-reference-points hypothesis using the findings in Mou and Zhou (2013). As is illustrated in Fig. 1A, three of the four targets were outside the circular cone array but were inside the wall in the study. The other target was inside both circles. The results showed that the impairment effect (i.e., the larger distance errors during testing than during learning) due to the removal of the wall during testing was negatively correlated with the number of the cones when this number was below 12; the impairment effect was eliminated when there were 18 or more cones. The shape-segment hypothesis could well explain these findings. According to this hypothesis, participants may divide the wall into equal segments/edges. This process is analogous to dividing the boundary of a clock into 


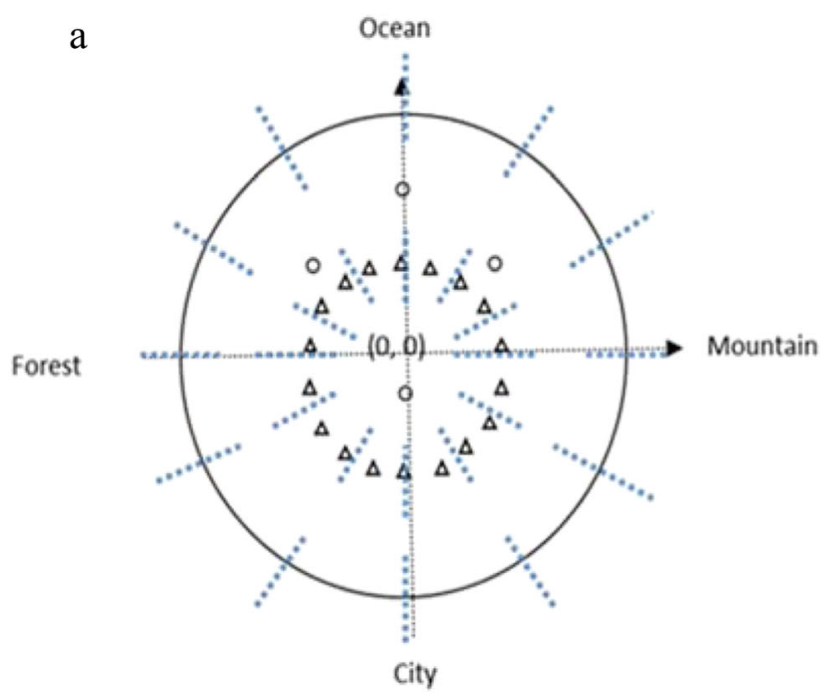

Fig. 1 Schematic diagram of segmentations based on the global shape of the environmental cues. The proposed environment segmentations are based on a circular wall (depicted as the circle) and a landmark array of multiple, evenly-spaced cones (depicted as the triangles). (A) Segmentations when the circular wall and the circular cone array shared the same center, as marked by the coordinate $(0,0)$. The dashed lines illustrate the hypothetical segmentations based on the two circles. The four target locations are depicted as the four dots. The four labels Ocean,

different hours (see Fig. 1A). Participants then differentiated the segments by using the orientation cues. This is similar to labeling each hour by a number (e.g., 12 o'clock). The location of a target was then encoded primarily relative to the closest segments. For example, the target location on the Ocean side (shown as the dot closest to the Ocean direction in Fig. 1A) can be specified by establishing a vector between the specific location and the wall segment pointing to the Ocean direction (e.g., $11.82 \mathrm{~m}$ inside the Ocean segment of the wall). When participants perceived the circle formed by the cones (18 or more cones), they also segmented this circular array and encoded a target location relative to the edge of the circle formed by the cones (e.g., the target location closest to the Ocean direction in Fig. 1A was $12.72 \mathrm{~m}$ outside the Ocean segment of the cone array).

It is important to note that the wall and the circle formed by the cone array shared the same center in Mou and Zhou's (2013) study. Therefore, the segments of the two circles were completely aligned (illustrated as the dashed lines in Fig. 1A). In particular, the distance between the corresponding local segments (segments in the same allocentric direction; e.g., the Ocean direction) of the two circles was the same across different directions. Specifically, the distance was equal to the radius difference between these two circles. If participants remembered the distance from a target to the edge of one circle, they should easily be able to calculate the distance from the same target to the corresponding edge of the other circle. For the target inside both circles, its distance to the wall edge minus its distance to the edge of the cone array was the radius

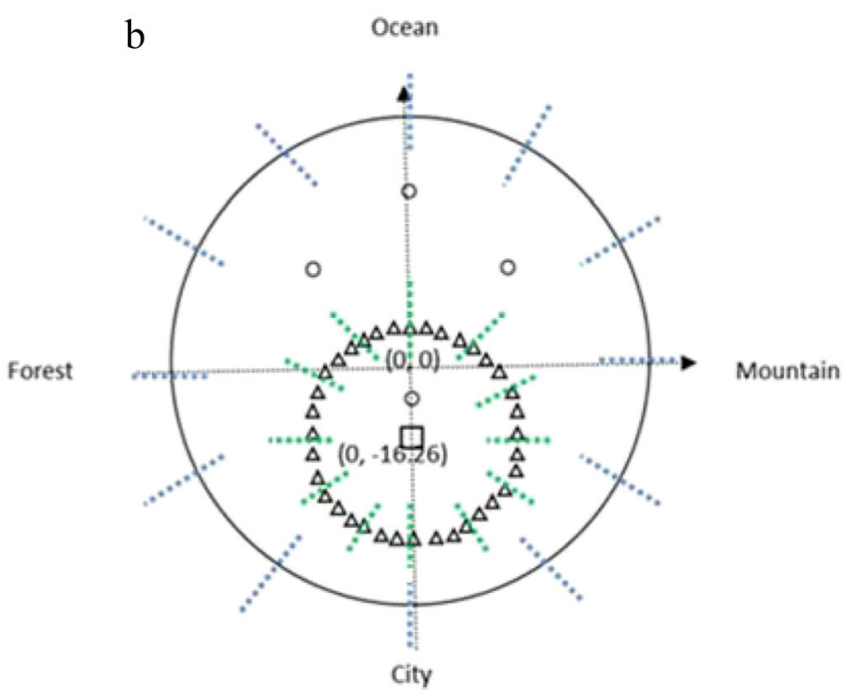

Mountain, City, and Forest illustrate the distal orientation cues. (B) Segmentations when the circular wall and the cone array are eccentric: The wall is centered at $(0,0)$, whereas the cone array is centered at $(0,-$ 16.26) (depicted as the square). The dashed lines on the outer circle illustrate the segmentation of the environment based on the wall cue, where as the dashed lines on the inner circle illustrate the segmentation of the environment based on the array cue

difference. For the other three targets, outside the cone array and inside the wall, the sum of the two distances was the radius difference.

Consequently, the vectors from one target to the edges of either circle can be computed on the basis of the vectors from the same target to the edges of the other circle. Therefore, the removal of the wall should not affect location memory if participants perceived the circle formed by the cones. With the decrease in the number of the cones (e.g., from 18 to 2), participants were less likely to perceive the circular shape of the cone array, and thus the cones were more likely to be treated as separate landmarks (discrete reference points). Lack of the segmentation information therefore led to the impairment effect in testing. As a result, the impairment effect due to the removal of the wall was observed, and the magnitude of such an effect increased when the number of the cones decreased. In addition, previous research has shown that the relative salience of discrete landmark cues and boundary cues has an impact on determining the attention resources devoted to the landmark/boundary and, hence, modulates the associative strength of the cue for learning (e.g., Buckley, Smith, \& Haselgrove, 2015; Kosaki, Austen, \& McGregor, 2013). Because a circular shape might be intrinsically more salient than discrete landmarks, participants were more likely to use the wall than to use the separate cones to specify the targets' locations during learning.

The sufficient-reference-points hypothesis can also explain the findings in Mou and Zhou's (2013) study, as long as we assume that the accuracy of object location memory would not 
decrease if at least 18 reference points were available to the participants during testing, regardless of the configuration formed by these reference points; otherwise, the accuracy of replacement performance decreased linearly when the number of the reference points (i.e., the cones) decreased.

In summary, the sufficient-reference-points hypothesis stipulates that the number of individual vectors is solely critical to accurate remembering of target locations, whereas the shape-segment hypothesis stipulates that the global shape information obtained from the configuration of the reference points is also important. Because the previous studies could not empirically distinguish between these two hypotheses, we distinguished between them in the present study.

In Experiment 1, we manipulated the configurations of the environmental cues, either forming an enclosure or not, while keeping the same number of reference points during testing. According to the sufficient-reference-points hypothesis, the number of individual reference points alone is critical to accurate recall of target locations. Therefore, we would not expect to see any effect from removing the global shape of the reference points, as long as a sufficient number of reference points remained during the tests. According to the shapesegment hypothesis, breaking the integrity of the shape information during testing would impair the segmentation information available to participants, thus impeding their accuracy in remembering during test.

In Experiment 2, we moved the center of the cone array so that the circles formed by the cones and the wall did not share the same center in the learning phrase. Either one of the circles was then removed during the testing phase. According to the sufficient-reference-points hypothesis, the number of individual reference points is critical to accurate encoding and recall of target locations. Therefore, we would not expect to see any impairment effect of removing either circle, since the remaining circle should provide a sufficient number of reference points. In contrast, according to the shape-segment hypothesis, the two eccentric circles would result in two different and independent segmentations, as the local edges from these two circles were not aligned (see Fig. 1B). Therefore, after learning the target locations respective to the two eccentric circular boundaries, participants would show impaired memory for object locations during testing when either circle was removed.

\section{Experiment 1}

The purpose of Experiment 1 was to examine whether environmental cues that provide an adequate number of reference points alone would be sufficient to eliminate the impairment effect in remembering goal locations due to removal of a wall. Alternatively, an enclosure and enough reference points might both be necessary to eliminating the impairment effect in remembering goal locations due to removal of the wall. The sufficient-reference-points hypothesis predicts the former finding, whereas the shape-segment hypothesis predicts the latter.

Four conditions were used to test these two possibilities. The purpose of the first condition was to replicate the previous finding that a landmark array of 18 evenly spaced cones could function as a boundary-like cue in encoding and subsequent remembering of object locations. We referred to this condition as ConeCirc-ConeCirc (standing for the "circular cone array" used in both the learning and testing phases; since the circular wall was presented during learning in all conditions throughout this article, we chose not to denote that cue in abbreviations of the conditions). During the learning phase, participants recalled the locations of four targets in the presence of both a circular wall and a cone array (Fig. 2A). The cone array consisted of 18 traffic cones that were distributed evenly in a circle-like configuration. During the testing phase, the wall was removed and the cone array was presented (Fig. 2B). On the basis of findings by Mou and Zhou (2013), we expected participants' performance for remembering object locations to be equivalent between the learning and testing phases in the ConeCirc-ConeCirc condition.

The cues presented during the learning phase were the same for the other three conditions (Fig. 2C), whereas the global shape information from the testing cues was manipulated across those conditions. During the learning phase, a landmark array containing two arcs of traffic cones was presented together with the circular wall. Each arc of the cone array comprised nine cones, and the central angle of each arc was $90^{\circ}$ (the angle subtended from the center to the two ends of the arc). This manipulation was designed to ensure that the landmark array provided the same number of reference points (and thus the same number of individual vectors) as the cone array (18) in the ConeCirc-ConeCirc condition.

Fig. 4 Top-view illustrations of the experimental environments in Experiment 1. (A) Learning phase in the ConeCirc-ConeCirc condition. The outside circle depicts the circular wall. The four dots specify the target locations (only one target was visible in each learning/testing trial of the actual experiments); the small triangles mark the landmark array. Both the circular wall and the landmark array were centered at $(0,0)$ (the coordinates were not visible during the experiment). The axes depicting the coordinate system specify the spatial structure of the experimental environment for readers but were not shown during the experiment. The four labels Ocean, City, Mountain, and Forest mark the orientation cues, set at infinite distances. The orientation cues were presented as pictures corresponding to the labels in the actual experiment. (B) Cue configuration during the testing phase in the ConeCirc-ConeCirc condition. (C) Environment of the learning phases in the ConeArc-ConeArc, ConeArc-WallArc, and ConeArc-ConeWallCirc conditions. (D) Cue configuration during the testing phase in the ConeArc-ConeArc condition. (E) Cue configuration during the testing phase in the ConeArcWallArc condition. The two arcs mark the two parts of the wall retained during testing. (F) Cue configuration during the testing phase in the ConeArc-ConeWallCirc condition 


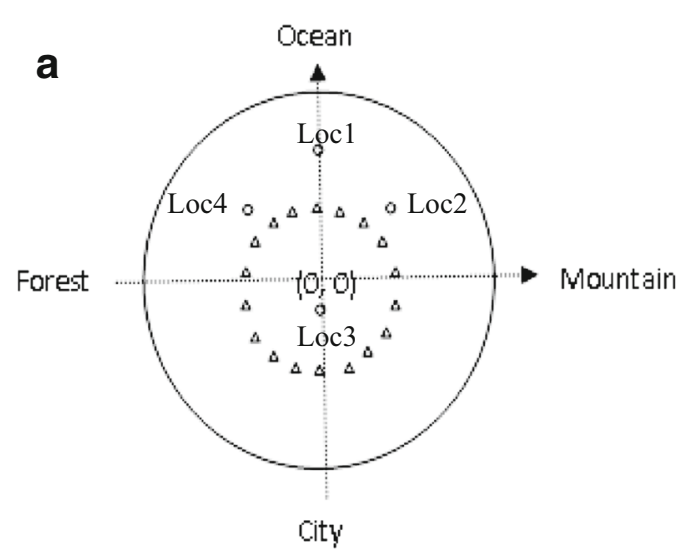

Learning of ConeCirc-ConeCirc

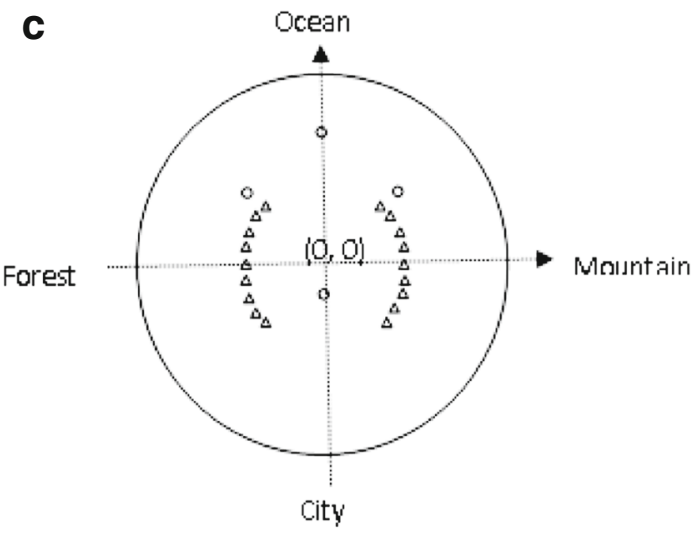

Learning of ConeArc-ConeArc,

ConeArc-WallArc, ConeArc-

ConeWallCirc

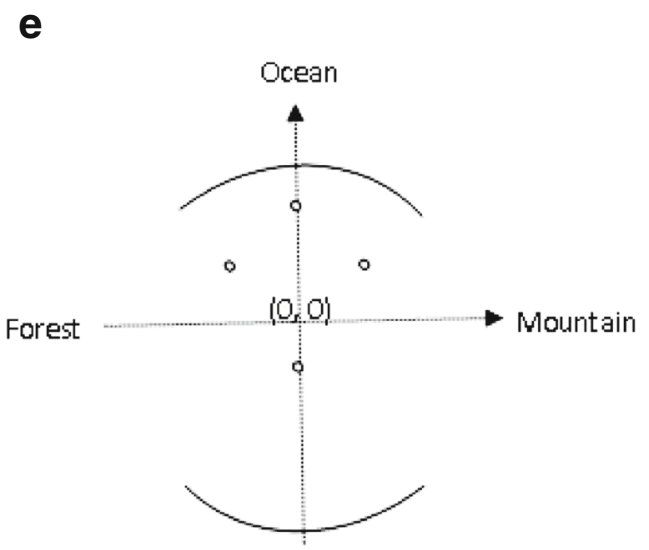

City

Testing of ConeArc-WallArc b

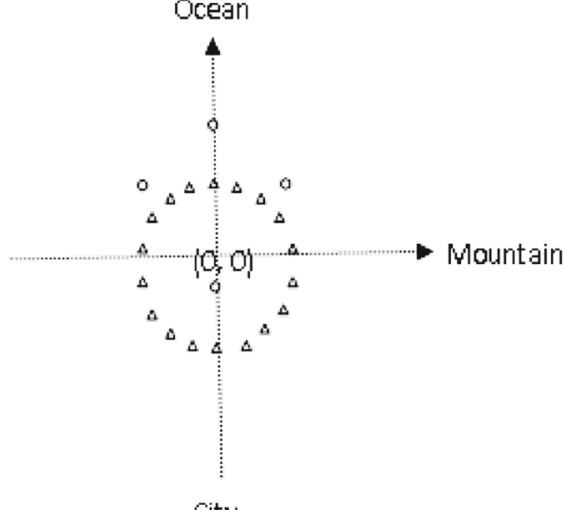

City

Testing of ConeCirc-ConeCirc

d Forest

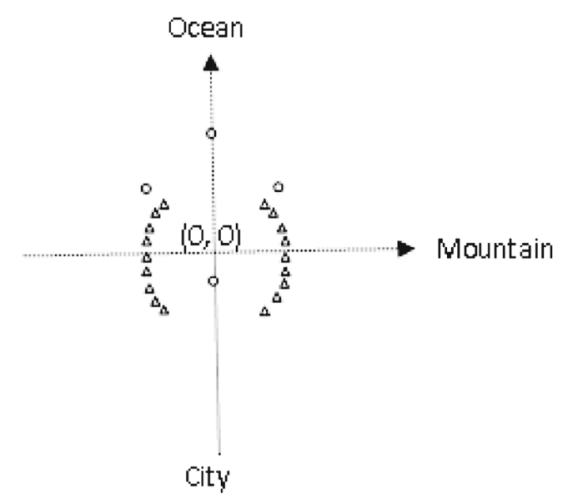

Testing of ConeArc-ConeArc

f

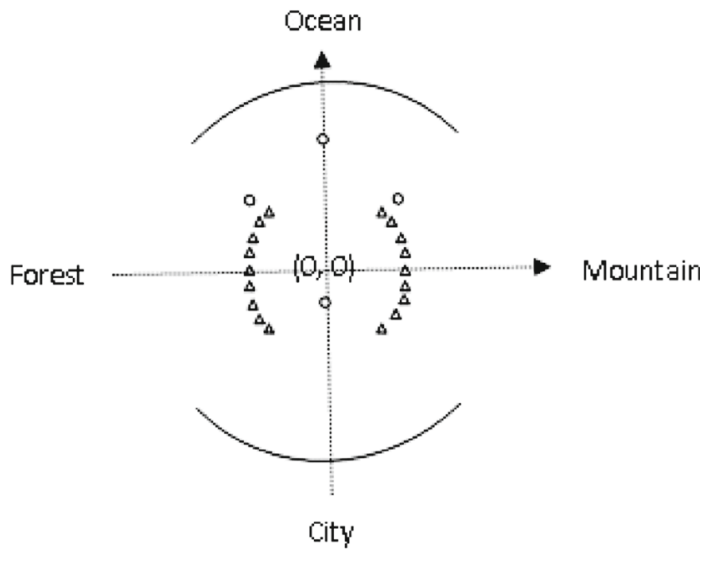

Testing of ConeArc-ConeWallCirc 
The cues presented during the testing phase differed among the last three conditions. In the ConeArc-ConeArc condition (standing for the "two-arc cone array" being used in addition to the wall in the learning phase, and the same cone array being used in testing phases; see Fig. 2C and D), the circular wall was removed and the two-arc cone array was presented during testing (Fig. 2D). In the ConeArc-WallArc condition (standing for the two-arc cone array being presented in addition to the wall during learning, and two wall arcs being presented during testing), two arcs of the circular wall were presented alone during testing (Fig. 2E). The central angle of each piece of the wall arcs (the angle subtended from the center to the two ends of the wall piece) was also $90^{\circ}$, and thus it was comparable to the cone arc in the ConeArc-ConeArc condition during testing. In the ConeArc-ConeWallCirc condition (standing for the two-arc cone array being used in addition to the wall during learning, and cone arcs and wall arcs together forming a circular shape being presented during testing), the two-arc cone array and the two arcs of the circular wall (the same as those used in the ConeArc-WallArc condition) were presented simultaneously during testing, so as to form a circular configuration (Fig. 2F). A contrast in the recall performance of object locations between the two conditions (ConeArc-ConeArc and ConeArc-WallArc) that lacked global shape information at retrieval and the two conditions (ConeCirc-ConeCirc and ConeArc-ConeWallCirc) with a complete global shape at retrieval would support the shapesegment hypothesis.

If multiple vectors are sufficient for encoding target locations and for retrieval from location memory, comparable performance would be expected between the learning phase and the testing phase (i.e., a null impairment effect from removing the wall during testing) in all four conditions, since at least 18 reference points remained in the testing phases of all conditions. According to Mou and Zhou (2013), 18 reference points should be sufficient to eliminate the impairment effect. Alternatively, if the circular shape information obtained from the configuration of the test cues is important in order for participants to induce the original segmentation, an impairment effect from removing the surface boundary would be expected in the ConeArc-ConeArc and ConeArc-WallArc conditions.

\section{Method}

Participants Seventy-two participants (36 men, 36 women) were recruited for participation from introductory psychology courses at the University of Alberta. The participants received partial course credit for their participation.

Material and design Participants learned four target locations in an immersive virtual reality environment. Orientation cues (Ocean, Forest, City, and Mountain) were set at an infinite distance on featureless grassland and were present throughout the entire experiment (see Fig. 3 for snapshots of the virtual environment). The main task was to place four objects (wood, lock, candle, and bottle) in their respective locations: $(0,38.18)$ [termed Loc1], (19.09, 24.75) [termed Loc2], (0, - 5.66) [termed Loc3], and $(-19.09,24.75)$ [termed Loc4] (the units are virtual meters [vm], marked as the dots in Fig. 2). The pairings between objects and locations were randomized across participants. Hence, the locations are referred to according to their coordinates rather than to the corresponding objects in our Analysis sections (the four locations are labeled in Fig. 2A). Participants completed four learning blocks (four trials per block, one trial per object within one block) in which feedback (object in its correct location) was given after each trial. Upon completing the learning phase, participants continued to the testing phase, which contained another four blocks of four trials without feedback. The sequence of objects within one block was randomized.

Participants were randomly assigned to the four conditions, consisting of different cue configurations. For the ConeArcConeArc condition, a circular wall $(50 \mathrm{vm}$ in radius, $1 \mathrm{vm}$ in height) and a circular array of 18 traffic cones were presented as the cues during learning (Fig. 2A). The two circles shared the same center $(0,0)$. Every two neighboring traffic cones in the cone array were $20^{\circ}$ of central angle (the cones are depicted as the triangles in Fig. 2A) apart from each other (the array had a radius of $25.46 \mathrm{vm})$. Participants were then tested with the cone array alone (Fig. 2B). The participants in the ConeArc-ConeArc condition were shown the circular wall and an 18-cone array in two arcs during learning. The circular wall was removed during testing, and the participants were shown the two-arc cone array alone. To create the two-arc cone array (illustrated as the triangles in Fig. $2 \mathrm{C}$ and $\mathrm{D}$; two neighboring cones were $10^{\circ}$ in central angle apart from each other, while the array had the radius of $25.46 \mathrm{vm}$ ), we first made a circular array comprising 36 traffic cones. Only the nine cones corresponding to the direction of the Mountain and the nine cones corresponding to the direction of the Forest were used. The central angle for each arc was $90^{\circ}$. The participants in the ConeArc-WallArc condition learned the target locations in the same cue configuration as those in the ConeArcConeArc condition. However, the ConeArc-WallArc group was tested with two parts of the circular wall alone (Fig. 2E). The two parts of the wall retained in this condition (depicted as the two arcs in Fig. 2E) were created by segmenting the wall into four equal arcs corresponding to the four orientation cues and extracting only the arcs in the Ocean and City directions. The central angle for each part of the wall was $90^{\circ}$. The learning phase in the ConeArc-ConeWallCirc condition was the same as that in the ConeArc-ConeArc and ConeArc-WallArc conditions (Fig. 2C). The cue configuration during testing of the ConeArc-ConeWallCirc condition was a combination of the test conditions in the ConeArc-ConeArc and the ConeArc-WallArc conditions - that is, both the two-arc cone array and the two parts of the wall were presented, to form a circular configuration (Fig. $2 \mathrm{~F})$. Eighteen participants were randomly assigned to each of the 
a

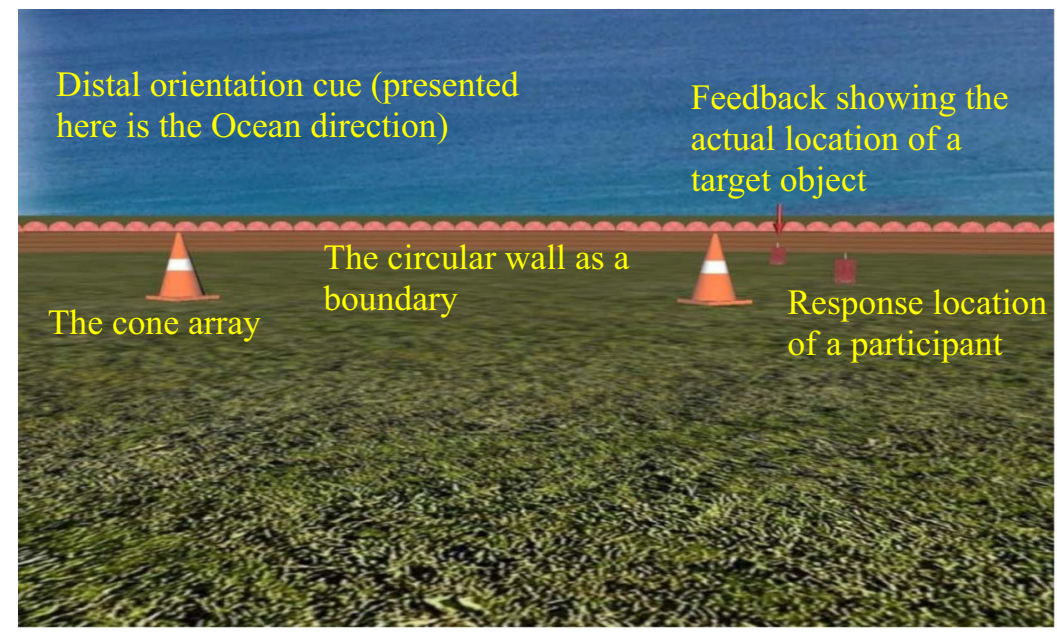

b

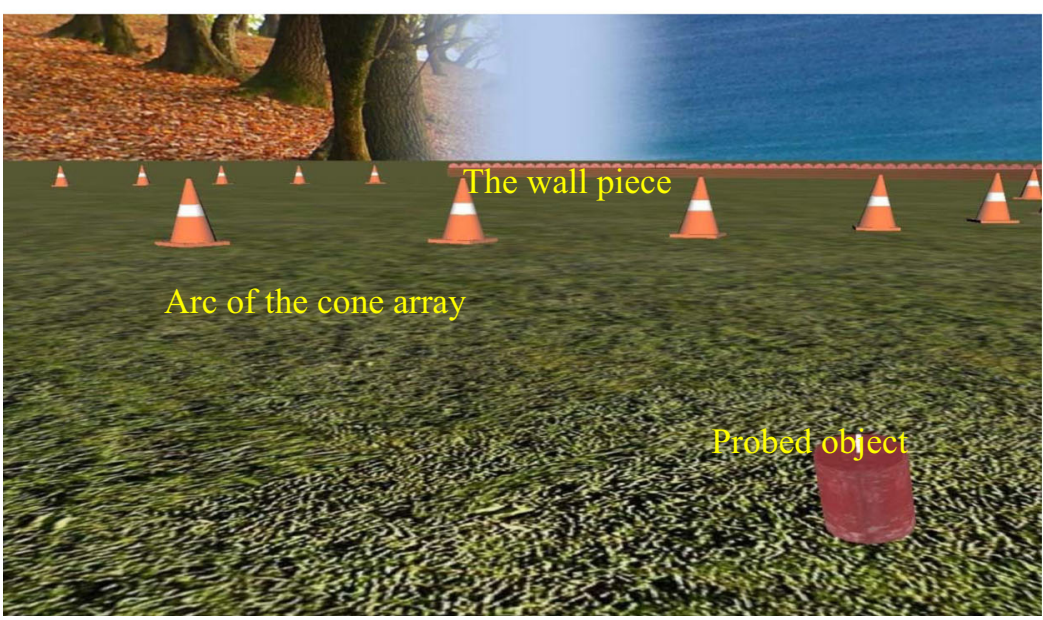

Fig. 3 Snapshots of the virtual environment in Experiment 1 from a first-person perspective. (A) The learning phase in the ConeCirc-ConeCirc condition. (B) The testing phase in the ConeArc-ConeWallCirc condition

conditions, with equal numbers of men and women in each condition.

The experiment was conducted in a physical room of $4 \times 4 \mathrm{~m}^{2}$; however, participants never saw the physical room throughout the experiment. A swivel chair was placed at the center of the room (which corresponded to the center of the circular wall and the circular cone array). Participants were seated in the chair during the experiment, and they could rotate the chair to change their orientations in the virtual reality environment.

The virtual environment was displayed using an nVisor SX60 head-mounted display (HMD; NVIS, Inc., Virginia). Graphics were rendered using the Vizard software (WorldViz, Santa Barbara, California). Participants' head orientation was tracked with an InterSense IS-900 motion-tracking system (InterSense, Inc., Massachusetts). Thus, through head rotation, participants could simply change their viewpoints. The height of the participants' viewpoint in the virtual environment was approximately $1.82 \mathrm{~m}$. Participants used a joystick to move forward and backward and to pick up and replace the objects in the environment.

The experiment used a mixed design with the experiment phase (learning vs. testing) as a within-subjects factor and the cue configuration (ConeCirc-ConeCirc, ConeArc-ConeArc, ConeArc-WallArc, and ConeArc-ConeWallCirc) as a between-subjects factor. The distances between the correct locations and the response locations given by participants were defined as the distance errors, which were used to measure participants' remembering of the target locations given by specific environment cues. As in our previous article (Mou \& Zhou, 2013), we compared participants' performance between the learning and testing phases to determine whether impairment effects were present. An impairment effect (greater distance errors during testing than during learning) would be expected if the remaining cue (the circular cone array, the two-arc cone array, the wall parts, or the combination of the wall parts and the cone array) was not sufficient for accurate recall of the target locations. 
Procedure After participants had read the instructions, they were blindfolded and led to the experiment room. Participants sat in the chair and donned the HMD. They were first instructed to look around the virtual environment and to identify the orientation cues, the landmark array, and the circular wall by changing their head orientation. They were then trained to use the joystick to move forward and backward and to pick up and drop objects. To become familiarized with the virtual reality environment, participants were required to move to the edge of the wall in each of the four directions indicated by the orientation cues. Participants were then instructed to collect the four target objects as they successively appeared on the grassland of the environment. Each object was only collected once. This was done to give participants a general idea about the to-be-learned targets and their locations.

Upon collection of all four targets, the learning phase started. Participants completed four blocks of four learning trials (one trial per target in each block). In each trial, one target appeared in the bottom right corner of the HMD screen, and participants attempted to place it in its original location. After their response, the same object was shown at its correct location, to provide feedback regarding response accuracy. Participants then navigated to the correct location to pick up the object and continued to the next trial. The testing phase began after the 16 learning trials. Participants then finished four blocks of four testing trials (one trial per target in each block) with the preassigned testing cues. Feedback was not given during the testing phase. At the beginning of each trial throughout the experiment, including both the learning and testing phases, participants were transported to start at a new random location (the starting locations were randomly picked within a circular area, the radius of which is $50 \mathrm{vm}$ ) and facing in a random direction. This was done to ensure that the target locations could not be specified by a fixed starting point or by the location of the target object probed in a previous trial.

\section{Results}

A data exclusion criterion was determined prior to data analysis: Any participant whose mean distance error during testing was greater than $100 \mathrm{vm}$ would be excluded, given that the radius of the wall was $50 \mathrm{vm}$. No participants were excluded in this experiment.

We measured distance errors (the distances between the correct locations and the response locations) as the dependent variable. There was a significant learning effect across the four learning blocks: Distance errors (averaged across the four objects in each block) decreased in subsequent blocks, $F(3,204)$ $=22.08, p<.001, \eta_{\mathrm{p}}{ }^{2}=.25$. No practice effects were observed during the testing phase: The distance errors in remembering object locations remained flat across the four testing blocks, $F(3,204)=2.07, p=.105, \eta_{\mathrm{p}}{ }^{2}=.03$. Hence, the distance errors in the last learning block indicated the acquisition of the location knowledge given by the corresponding learning cues, whereas distance errors averaged across the four testing blocks indicated the memory of the target locations relative to the corresponding testing cues. The learning effect across the four learning blocks and the lack of a practice effect at test were also observed in Experiment 2. Therefore, in both experiments we analyzed the distance errors in the last learning block and the distance errors averaged across the four testing blocks.

Figure 4 displays the mean distance errors as a function of cue configuration (ConeCirc-ConeCirc, ConeArc-ConeArc, ConeArc-WallArc, and ConeArc-ConeWallCirc conditions) and the experimental phase (the last learning block vs. all testing blocks). As is shown in Fig. 4, there was a significant increase in the magnitude of the distance errors during the entire testing phase as compared to the last learning block in the ConeArc-ConeArc and ConeArc-WallArc conditions; this increase indicates an impairment effect in both groups upon removal of the partial cues. However, no impairment effect was observed in either the ConeCirc-ConeCirc or the ConeArc-ConeWallCirc conditions. Further analysis with mixed-model analyses of variance (ANOVAs) confirmed this observation, with cue configuration (ConeCirc-ConeCirc, ConeArc-ConeArc, ConeArc-WallArc, and ConeArcConeWallCirc) as a between-subjects variable and experimental phase as a within-subjects variable.

The main effect of experimental phase was significant, $F(1$, 68) $=14.54, p<.001, \eta_{\mathrm{p}}{ }^{2}=.18$, but a main effect of cue configuration was not evident, $F(3,68)=0.59, p=.62, \eta_{\mathrm{p}}{ }^{2}$ $=.03$. The interaction between cue configuration and experimental phase was significant: $F(3,68)=3.59, p=.02, \eta_{\mathrm{p}}{ }^{2}=$ .14 .

Planned comparisons (using a two-tailed $t$ test) revealed greater distance errors during the testing phase than during the learning phase in both the ConeArcConeArc condition, $t(68)=4.09, p<.001$, and the ConeArc-WallArc condition, $t(68)=2.72, p=.008$. However, distance errors did not increase in magnitude in either the ConeCirc-ConeCirc condition, $t(68)=$ $1.07, p=.29$, or the ConeArc-ConeWallCirc condition, $t(68)=0.25, p=.81$. A one-way ANOVA on the distance errors in the last learning block confirmed that there was no difference in participants' performance toward the end of learning phase across the four cue configuration conditions, $F(3,68)=0.52, p=.67, \eta_{p}^{2}$ $=.02$. The lack of a difference in learning performance indicated that the impairment observed in the two conditions in which the global circular shape was disrupted was not due to do insufficient learning. 


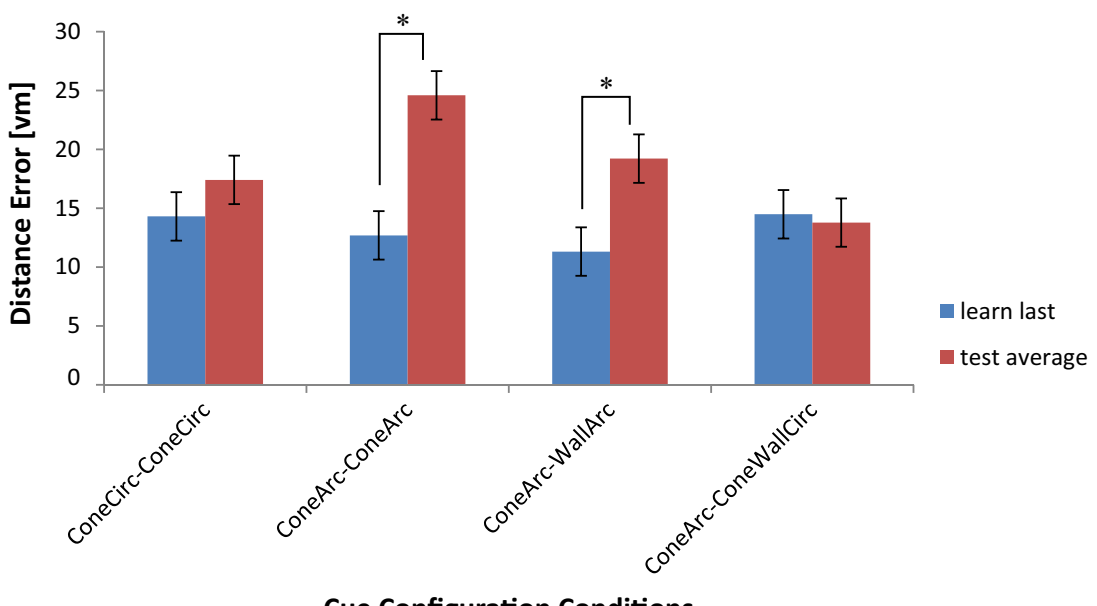

Fig. 4 Distance errors as a function of cue configuration and experimental phase in Experiment 1 (error bars show \pm 1 standard errors after removing between-subjects variation)

According to the shape-segment hypothesis, segments closer to the target locations could be selected as the reference points for encoding individual locations, so we would expect that removing different parts of the cone array or the wall might have differential effects on memory performance at different target locations. That is, for instance, removing the wall parts facing the ocean direction might exert more of an impairment on location memory for Loc1 (Fig. 2A and D). Therefore, in addition to experimental phase and cue configuration, we also included location identity (i.e., Loc1-Loc4) as a within-subjects variable in a three-way factorial analysis, to further examine whether location memory differed for $\mathrm{a}$

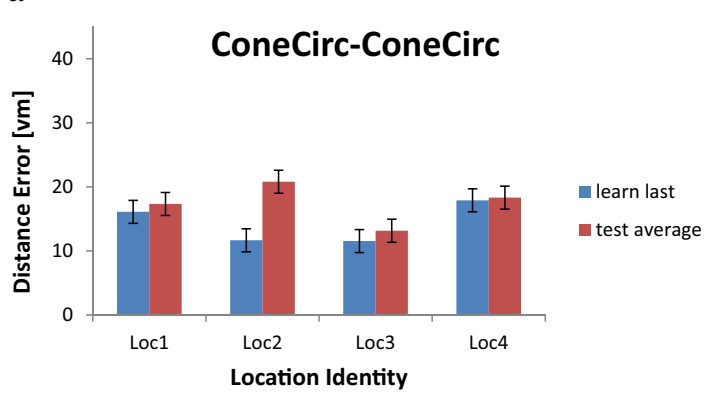

c

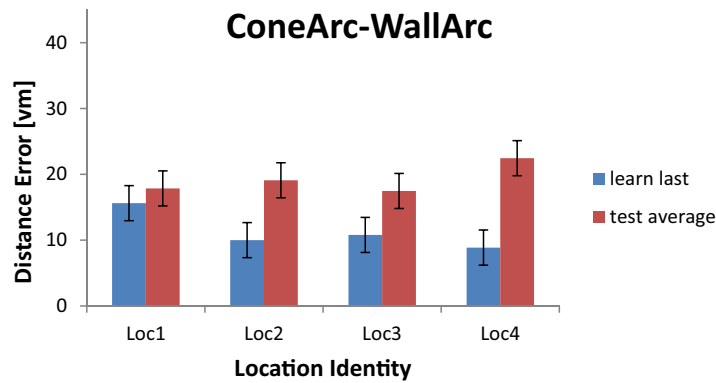

Fig. 5 Distance errors as a function of location identity and experimental phase across the four cue configuration conditions in Experiment 1 (error bars show \pm 1 standard errors after removing between-subjects variation).

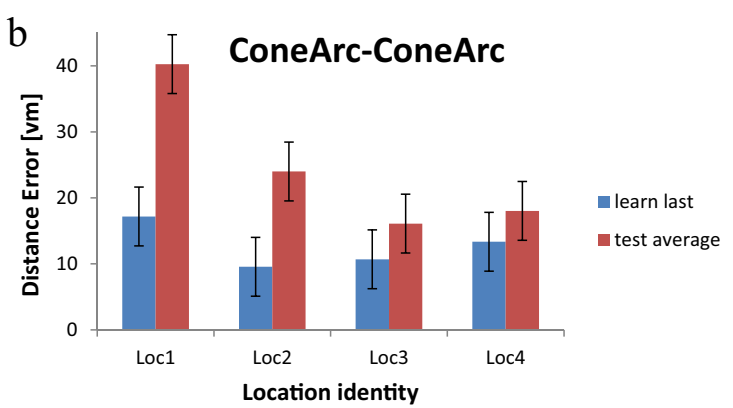

d

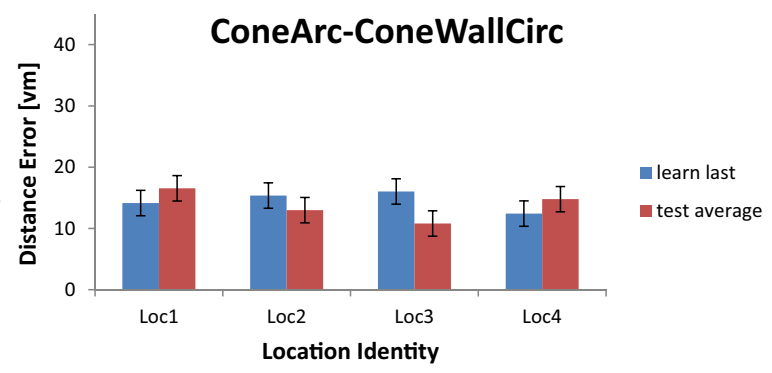

(A) The ConeCirc-ConeCirc condition. (B) The ConeArc-ConeArc condition. (C) The ConeArc-WallArc condition. (D) The ConeArcConeWallCirc condition 
different target locations depending on the cue configuration during testing .

Our analysis revealed a significant three-way interaction among experiment phase, location identity and the testing cue configuration, $F(9,204)=2.05, p=.036, \eta_{\mathrm{p}}{ }^{2}=.08$. We conducted four separate two-way ANOVAs examining the interaction of experiment phase and location identity in all testing cue conditions (Fig. 5). The interaction of experiment phase and location identity was not significant in any of the four conditions: ConeCirc-ConeCirc, $F(3,51)=2.55, p=.07$, $\eta_{\mathrm{p}}{ }^{2}=.13$; ConeArc-ConeArc, $F(3,51)=1.90, p=.14, \eta_{\mathrm{p}}{ }^{2}=$ .10 ; ConeArc-WallArc, $F(3,51)=1.80, p=.16, \eta_{\mathrm{p}}{ }^{2}=.10$; and ConeArc-ConeWallCirc, $F(3,51)=1.65, p=.19, \eta_{\mathrm{p}}{ }^{2}=.09$. A memory decrease during testing was observed for all four locations in the ConeArc-ConeArc, $F(1,17)=5.20, p=.04$, $\eta_{\mathrm{p}}{ }^{2}=.23$, and ConeArc-WallArc, $F(1,17)=22.25, p<.001$, $\eta_{\mathrm{p}}{ }^{2}=.57$, conditions. In contrast, no decrease in object location memory was observed in the ConeCirc-ConeCirc, $F(1$, $17)=3.82, p=.07, \eta_{\mathrm{p}}{ }^{2}=.18$, or ConeCirc-ConeWallCirc, $F(1,17)=0.40, p=.54, \eta_{\mathrm{p}}{ }^{2}=.02$, conditions, in which the global shape segmentation was intact during testing.

\section{Discussion}

The results of Experiment 1 support the shape-segment hypothesis rather than the sufficient-reference-points hypothesis. According to the shape-segment hypothesis, the global shape of the circular wall affected the selection of reference points for encoding target locations. In particular, this global circular shape together with the distal orientation cues enabled participants to differentiate the segments of the wall. Participants further encoded a given location with respect to the differentiated segments. A disruption of global shape integrity during testing would likely impede recovery of the original segmentation acquired from learning, as we can see from the impaired performance in the ConeArc-ConeArc and ConeArcWallArc conditions. By contrast, the participants in the ConeArc-ConeWallCirc and ConeCirc-ConeCirc conditions were able to recover the original circular shape (e.g., by integrating the wall parts and the cone arcs in the ConeArcConeWallCirc condition), demonstrating intact location memory during testing. These findings challenge the sufficientreference-points hypothesis, which stipulates that only the number of the reference points during testing is critical for accurate recall of target locations. According to this hypothesis, a null impairment effect would be observed in any of these conditions, since the number of reference points during testing was sufficient (i.e., 18 or more); our results, however, were inconsistent with this prediction.

\footnotetext{
${ }^{1}$ We thank the two anonymous reviewers for suggesting this further analysis to examine the differential effects of cue configuration during testing on different target locations.
}

Further analysis of the differential effects of cue configuration on memory performance for different target locations revealed no interaction effect between experiment phase and location identity in any of the four cue configuration conditions. This result again demonstrates that the global shape formed during testing was critical for recalling the target locations: A disruption in recovering the segmentation during testing interfered with object location memory.

In addition to providing reference points for encoding locations, a boundary also provides scale information for objects residing within the enclosure. One possible explanation for the decreased performance during testing of the ConeArc-ConeArc and ConeArc-WallArc conditions is that the absence of a complete wall also eliminated this scale information. Note that in the ConeCirc-ConeCirc condition, three of the four targets were outside the circular cone array. Participants were able to retain their object location memory without the outmost boundary, suggesting that they were able to encode the distance information from the inner cone circle.

\section{Experiment 2}

Experiment 2 was designed to provide further evidence for the shape-segment hypothesis. Participants learned four target locations relative to two eccentric circles, one formed by a continuous wall and the other formed by a cone array. Participants were subsequently tested (by replacing the objects in their original locations) when either circle was removed. The question was whether an impairment effect (decreased object location memory during testing) would be observed due to the removal of one circle. The number of the cones was 36 (a 36-cone array), which should be sufficient to eliminate the impairment effect upon the removal of the wall. This lack of an impairment effect was demonstrated in Mou and Zhou's (2013) study, as well as in Experiment 1 of the present study, in which the cone array (containing 18 or more cones) was concentric with the wall. The shape-segment hypothesis predicts that an impairment effect would emerge upon removal of either circle, because the two eccentric circles would produce two independent segmentations (e.g., Fig. 1B), and participants might not be able to simultaneously utilize both. In contrast, the sufficient-reference-points hypothesis predicts no impairment effect upon removal of either circle, because the number of the reference points during testing should be sufficient.

\section{Method}

Participants Forty-nine undergraduate students (24 men, 25 women) were recruited for participation from an introductory psychology course of the University of Alberta. One female 


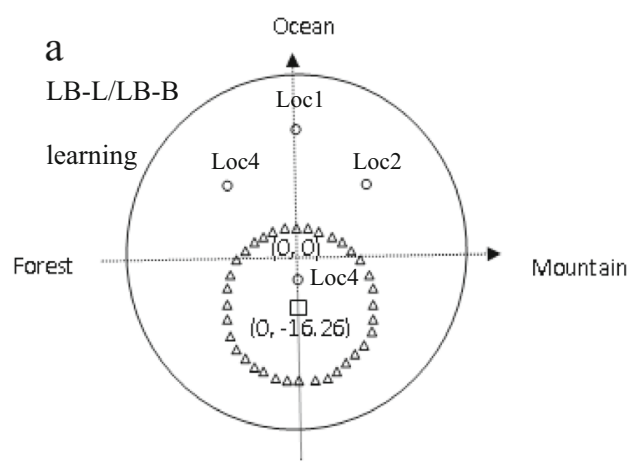

City

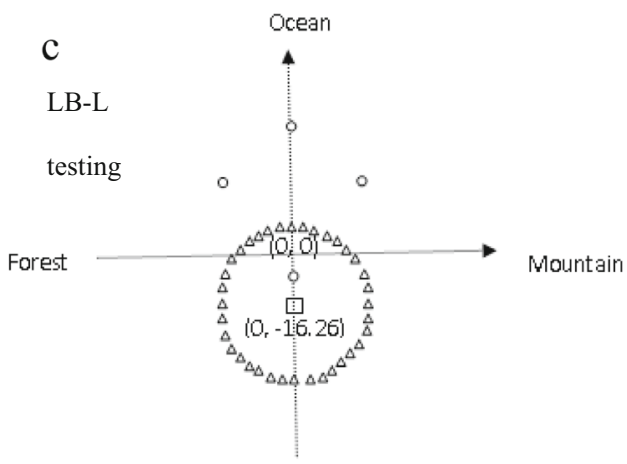

City

Fig. 6 Top-view illustrations of the experimental environment in Experiment 2. (A) The learning environment in both the LB-L and LB-B conditions. The square marks the center of the cone array, which

participant was excluded from the sample because her mean distance error across the four test blocks was greater than 100 $\mathrm{m}$. All participants received partial credit for their participation.

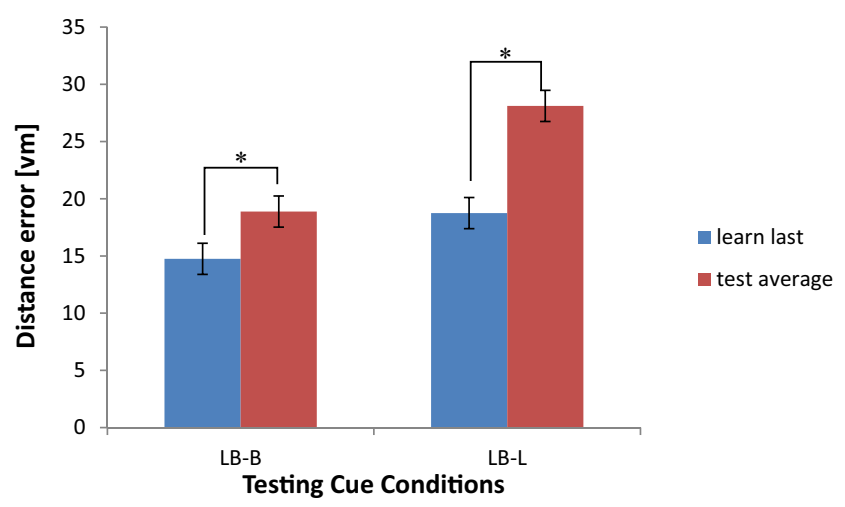

Fig. 7 Distance errors as a function of the testing cue and experimental phase in Experiment 2 (error bars show \pm 1 standard errors after removing between-subjects variation). LB-B, learning with both the wall and the shifted cone array, and testing with the wall; LB-L, learning with both the wall the shifted cone array, and testing with the cone array

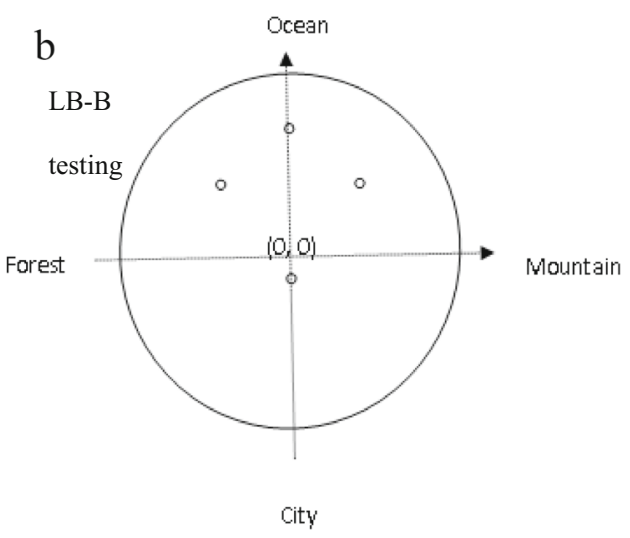

was not displayed during the actual experiment. (B) Testing environment in the LB-B condition. (C) Testing environment in the LB-L condition.

Material, design, and procedure The center of the 36-cone array (in which the cones were evenly spaced, as depicted by the triangles in Fig. 6A and C) was located at the coordinate $(0,-16.26)$ (illustrated as the square in Fig. $6 \mathrm{~A}$ and $\mathrm{C})$, so that the wall and the cone array were eccentric. The participants were randomly assigned to two groups, with 24 participants (12 men, 12 women) in each group. Both groups learned the target locations with two cues presented (both the circular wall and the circular cone array). The boundary group (referred to as $L B-B$ ) was tested with only the circular wall (Fig. 6B), whereas the landmark group (referred to as $L B-L$ ) was tested with only the cone array (Fig. 6C). The orientation cues were presented during the entire experiment. The rest of the design and the procedure were identical to those in Experiment 1.

\section{Results and discussion}

Figure 7 displays the mean distance errors plotted as a function of the experimental phase (the last learning block vs. the average of the four testing blocks) and the testing cue (LB-B vs. LB-L). As is shown in Fig. 7, impairment effects were observed in both conditions. Repeated measures ANOVAs 
using testing cue as a between-subjects variable and experimental phase as a within-subjects variable confirmed this observation.

The main effect of experimental phase was significant, $F(1$, 46) $=24.56, p<.001, \eta_{\mathrm{p}}{ }^{2}=.35$, and the main effect of testing cue was also significant, $F(1,46)=9.05, p=.004, \eta_{\mathrm{p}}{ }^{2}=.16$. The interaction between these two variables was not significant: $F(1,46)=3.72, p=.06, \eta_{\mathrm{p}}{ }^{2}=.08$. Planned comparisons (two-tailed $t$ tests) revealed greater distance errors during testing than during the fourth learning block in both the LB-B, $t(46)=2.14, p=.04$, and LB-L, $t(46)=4.87, p<.001$, conditions.

These results indicated that the removal of either the circular wall or the cone array impaired memory retrieval of the target locations, which was consistent with the prediction based on the shape-segment hypothesis but not on the sufficient-reference-points hypothesis.

Although the interaction effect was not significant $[F(1,46)$ $\left.=3.72, p=.06, \eta_{\mathrm{p}}{ }^{2}=.08\right]$, Fig. 7 shows that the larger distance errors in the LB-L than in the LB-B group might occur primarily during testing. Note that the LB-L and LB-B groups were are identical in the learning phase, so we should not expect any difference in distance errors in the learning phase. A two-sample $t$ test was carried out to examine the distance errors (averaged across the four probed locations) between the two conditions. Participants displayed equivalent learning performance for location memory in the two conditions, $t(46)=-$

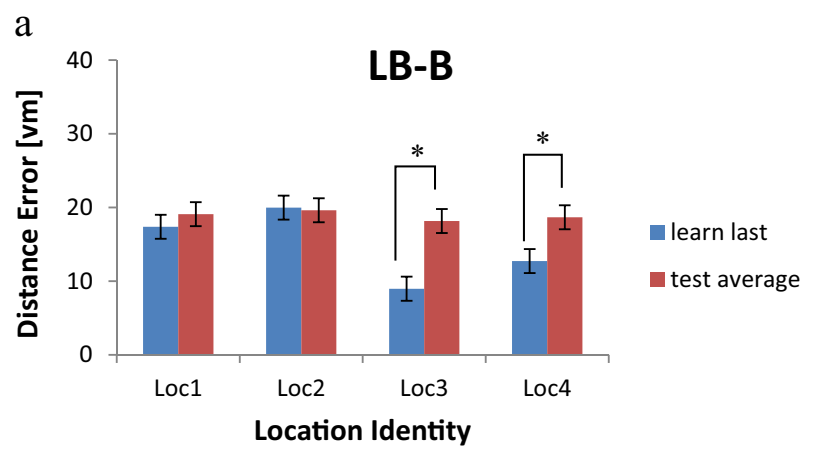

b

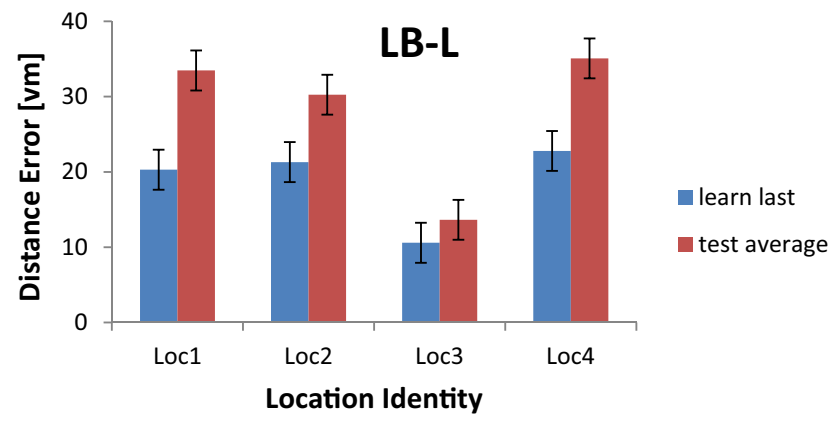

Fig. 8 Distance errors as a function of location identity and experimental phase between the two testing-cue conditions in Experiment 2 (error bars show \pm 1 standard errors after removing between-subjects variation). (A) LB-B. (B) LB-L
$1.79, p=.08$. Thus, the main effect of testing cue (whether the cone array or the wall alone was presented during testing) was driven mainly by the more pronounced decrease in location memory in the LB-L than in the LB-B group. One explanation could be that when participants used both the wall and the shifted cone array to encode object locations independently, these two cues competed. The shifted cone array might have been a less effective cue than the wall because (a) the cone array was smaller than the wall and (b) the shifted cone array overall was farther away from the target locations than was the wall, if we assume that people prefer a more stable and also a closer cue. Hence, we found a larger impairment effect from removing the wall than from removing the cone array. Note that the participants in Experiment 1 (ConeArc-ConeArc) had used both the wall and the concentric cone array to encode object locations dependently. Hence, removal of either cue did not affect remembering of the target locations.

We conducted a further analysis to examine whether the testing cues exerted differential effects on location memory performance for different probed locations. A three-way ANOVA was conducted with testing cue as a betweensubjects variable and experimental phase and location identity (i.e., Loc1-Loc4) as within-subjects variables. A three-way interaction was revealed, $F(3,138)=3.20, p=.025, \eta_{\mathrm{p}}{ }^{2}=$ .065 . Two separate two-way ANOVAs examined the interaction of experimental phase and location identity for the LB-B (Fig. 8A) and LB-L (Fig. 8B)' conditions, respectively. This interaction was significant only for $\mathrm{LB}-\mathrm{B}, F(3,69)=3.44, p=$ $.02, \eta_{\mathrm{p}}{ }^{2}=.13$, but not for LB-L, $F(3,69)=1.50, p=.22, \eta_{\mathrm{p}}{ }^{2}=$ .06 . Specifically, paired-sample $t$ tests revealed a decrease in memory performance for Loc3, $t(23)=3.98, p<.001$, and for Loc4, $t(23)=3.28, p=.002$, whereas memory performance was retained for Loc1, $t(23)=0.74, p=.23$, and Loc2, $t(23)=$ $0.16, p=.44$. This result indicates that the reference points available in the environment were not treated equally. The shape-segmentation process enabled participants to differentiate the available reference points (e.g., wall parts or cones closer to the Ocean direction in Fig. 6A), and subsequently they chose relevant reference points for encoding a specific target (e.g., the wall part closer to Loc1). Removing these more informative reference points would impair the corresponding location memory (e.g., decreased memory for Loc3 and retained memory for Loc1 when only the wall was presented in LB-B). In contrast, the sufficient-referencepoints hypothesis would imply that all reference points would be treated equally.

Note that in the present project, we only contrasted the shape-segment hypothesis and the sufficient-reference-points hypothesis. A distance-based hypothesis, claiming that participants only selected reference points on the basis of their distances from individual targets could also explain the present data. For example, the object in the center (Loc3) was closer to the cone array (shortest distance $=14.86 \mathrm{vm}$ ) than to the 
boundary (shortest distance $=44.34$ ), so people used the cones to encode this object. We acknowledge that this hypothesis can explain the present data (e.g., the impairment effect for Loc3 in LB-B). However, this hypothesis was not consistent with the finding of Mou and Zhou (2013). In their Experiment 2, participants learned the same four locations relative to a cone array and a wall as in the ConeCirc-ConeCirc condition here. The distance-based hypothesis predicts that removal of the cone array in the test phase would produce an impairment effect, because at least three locations would be encoded relative to the cone. However, Mou and Zhou's Experiment 2 did not show any impairment effect due to removal of the cones. In addition, a smaller impairment effect was also observed for Loc 4 in the present LB-B condition, whereas memory for all four locations was impaired in the ConeArc-ConeArc and ConeArc-WallArc conditions, regardless of the distance between the probed location and the removed wall/array parts. Therefore, such a distance-based hypothesis was also inconsistent with the findings of the present Experiment 1.

\section{General discussion}

In this study, we investigated the role of the global shape of a boundary in encoding and remembering the locations of target objects. In particular, we distinguished between the shapesegment hypothesis and the sufficient-reference-points hypothesis, derived from the vector-based localization models (Cheng, 1989; Mou \& Zhou, 2013; O'Keefe \& Burgess, 1996). The shape-segment hypothesis acknowledges the importance of the global shape formed by multiple reference points to localization. However, the sufficient-referencepoints hypothesis emphasizes the importance of the number of individual reference points to localization, but it overlooks the importance of the global configuration formed by the reference points. Our findings favor the shape-segment over the sufficient-reference-points hypothesis.

The sufficient-reference-points hypothesis derives from vector-based models, including the BVC model (O'Keefe \& Burgess, 1996), the multiple-reference-point hypothesis (Mou $\&$ Zhou, 2013), and the vector-sum model (Cheng, 1989), which focus on the importance of the individual vectors established between a target location and a number of reference points provided by an environmental cue. According to the sufficient-reference-points hypothesis, having a larger number of reference points to provide more individual vectors will contribute to an increasingly accurate representation of a target location, until a sufficient number of reference points has been obtained (i.e., 18 or more cones; Mou \& Zhou, 2013). Therefore, accuracy in the remembering of target locations does not decrease when some reference points are removed, as long as the total number is sufficient during testing. By contrast, according to the shape-segment hypothesis, apart from these individual vectors, presenting a larger number of discrete reference points also leads to a higher likelihood that people will perceive the shape formed by these reference points. When people perceive such shape information, they segment the shape into local parts, differentiate those parts using orientation cues, and encode a given target location relative to the differentiated segments. A similar shapesegment process occurs during testing, to reidentify the original reference points used in the learning phase. Therefore, object location memory decreases upon the removal of some reference points when this removal impairs recovery of the original segmentation.

In Experiment 1 we showed that the global shape formed by the testing cues was critical in eliminating the impairment effect that resulted from removing the wall or parts of the wall that were presented during learning, favoring the shapesegment hypothesis. Four groups of participants (ConeCircConeCirc, ConeArc-ConeArc, ConeArc-WallArc, and ConeArc-ConeWallCirc; see Fig. 2) learned four locations relative to both a cone array and a circular wall. Although equal numbers of cones (18) were available during learning in all four conditions, the cone array in the ConeCircConeCirc group formed a circle, whereas the arrays in the other three conditions did not. Participants were tested subsequently with the landmark array alone (in the ConeCircConeCirc and ConeArc-ConeArc conditions), with two parts of the wall that maintained the same visual angle as the two parts of the noncircle array (the ConeArc-WallArc condition), or with a combination of wall parts and an array that together formed a circular shape (the ConeArc-ConeWallCirc condition). Poorer object location memory in the testing phase than in the learning phase (i.e., an impairment effect due to the removal of the corresponding environmental cues) was observed when the configurations of the testing cues were not consistent with the original shape of the wall (i.e., a circular shape), but spared recall performance was observed when the global configurations of the testing cues echoed the original shape of the wall.

This finding was consistent with the shape-segment hypothesis. According to this hypothesis, disruption of the global shape during testing of the ConeArc-ConeArc and ConeArc-WallArc conditions (where a circular configuration was always provided by the wall during learning) would likely disrupt recovery of the original segmentation, causing an impairment effect in retrieving the location memory. In contrast, our findings challenged the sufficient-reference-points hypothesis. It is hard for this hypothesis to explain why the global shape during testing should matter (e.g., the contrast between ConeArc-ConeWallCirc and ConeArc-ConeArc/ ConeArc-WallArc). Furthermore, the sufficient-referencepoints hypothesis also has difficulty explaining why there was impairment effect in the ConeArc-ConeArc condition but not in the ConeCirc-ConeCirc condition (Fig. 2). 
According to the sufficient-reference-points hypothesis, because there were equal amounts of reference points during testing in the ConeArc-ConeArc and ConeCirc-ConeCirc conditions, there should have been no impairment effect in the ConeArc-ConeWallCirc condition, as there had been no impairment effect in the ConeCirc-ConeCirc condition.

Experiment 2 showed an impairment effect in object location memory due to removal of either the circular wall or the cone array during testing when the wall and the circle formed by the cone array had been eccentric during learning. This finding was again consistent with the prediction of the shape-segment hypothesis. According to this hypothesis, two concentric circles create two completely aligned segmentations (Fig. 1A). Furthermore, the corresponding segments (aligned in allocentric directions) in the two circles have a fixed distance between them (i.e., the radius difference) across all directions. Therefore, the vector from a target to the segment of one of the circles can be used to infer the vector to the corresponding segment of the other circle. Hence, the removal of either circle during testing should not affect location memory. Conversely, two eccentric circles would create two independent segmentations (Fig. 1B). A competition could occur between the two segmentations; hence, an inability to use both cues would be expected. Consistent with this prediction, the impairment effect was observed when either circle was removed during testing. Our finding, however, was inconsistent with the prediction of the sufficient-reference-points hypothesis. According to that hypothesis, as long as there were enough reference points during testing, irrespective of whether the circle formed by the landmark array was concentric or eccentric with the wall, removing the wall should not impair performance in recall of the learned object locations. The number of the reference points during testing in Experiment 2 should have been adequate for recovering the location memory, as was suggested in Experiment 1 of the present study (ConeCirc-ConeCirc condition) as well as in Mou and Zhou (2013). Therefore, the sufficient-reference-points hypothesis would not predict any impairment effect in Experiment 2.

In Experiment 2, we observed that the testing cues/ configuration elicited differential impairment effects on the probed locations, depending on the distance between the removed parts and the target locations (Loc3 and Loc4 in LBB). The shape-segment hypothesis could explain this differential effect, because the global shape/configuration provides a way for participants to segment the reference points. For example, a circular shape can be divided into 12 directions (like a clock face), and 12 reference points could be segmented from the circular enclosure. Then, each target would be encoded with respect to the closest reference point rather than to all 12 of the reference points. In contrast, the sufficientreference-points hypothesis implies that all reference points would be treated equally, so that each might be used for encoding the target location. Therefore, according to the shape-segment hypothesis, removing reference points in specific directions during testing would impair location memory for the locations that had been encoded relative to the removed reference points. Different impairment effect would be observed for different target locations.

The shape-segment hypothesis, as opposed to the sufficient-reference-points hypothesis, underscores one important difference between a boundary cue and a single landmark cue in goal localization. According to the sufficientreference-points hypothesis (see also the multiple-referencepoint hypothesis proposed by Mou \& Zhou, 2013), a surfacebased boundary or a landmark array forming a certain geometric shape could provide multiple vectors to encode a target location, whereas a single object-based landmark can only provide a single vector. Thus, the difference between a landmark and a boundary might be quantitative: single versus multiple vectors. According to the shape-segment hypothesis, the difference might also be qualitative, in that the global shape knowledge extracted from the configuration of multiple reference points could be regarded as distinctive from the elemental knowledge of a single vector.

By emphasizing the importance of global shape information in a location memory task, the shape-segment hypothesis also links the goal localization literature to the reorientation literature, which has already established the importance of global shape information in reorientation. The difference between reorientation tasks and object location memory tasks might not be as significant as it appears (see, e.g., Burgess, 2008; Lew, 2011). From the perspective of the shape-segment hypothesis, the first stage during the encoding of a target location (i.e., segmenting a shape into parts and differentiating the parts by orientation cues) might also be applied in a reorientation task (Miller \& Shettleworth, 2007). In a typical reorientation task, participants learn a target placed at one corner of a rectangular room that also contains some unique features to distinguish the correct corner from its diagonal counterpart. After disorientation, participants search for the target (e.g., Cheng, 1986). According to the shape-segment hypothesis, when participants learn a target location, they might possibly segment the room into four corners and four walls. They would then use the geometry information (e.g., the principal axis; Gallistel, 1990) to distinguish between two geometrically different pairs of corners: the pair on the left side of the body versus the pair on the right side of the body, when the body is aligned with the principal axis of the room. Therefore, during testing, people can still distinguish between these two pairs of corners by relying on the geometry information of the room and can search at the correct corner rather than the diagonal (geometrically identical) corner. Such use of abstract geometric information (including distance and sense of direction) seems to developed at an early age, because 2-year-old children are already able to reorient themselves on the basis of the 
distance and direction information extracted from room geometry (e.g., Lee, Sovrano, \& Spelke, 2012).

Reorientation tasks and tasks probing object location memory might also share similar cognitive mechanisms when participants select between a continuous boundary and a single landmark as their primary spatial cue. The adaptivecombination theory was proposed to explain why participants ignored featural cues that in principle would help them avoid the diagonal corner in reorientation tasks (Newcombe \& Ratliff, 2007; Ratliff \& Newcombe, 2008). This theory speculates that although both a landmark and the shape based on a boundary can be used to reorient, the cue selected depends on the relative saliences of the cues; since in general a shape is more salient than a landmark, people are more likely to ignore the featural cues. The neural underpinnings of such reliance on global geometry to regain one's sense of direction were also identified in a recent electrophysiological study (Keinath, Julian, Epstein, \& Muzzio, 2017). In accordance with the adaptive-combination theory and the empirical evidence, the shape-segment hypothesis also suggests that both a shape and a landmark can be primarily used in goal localization. The cue selection process will depend on the relative saliences of the cues, and thus, people tend to use the shape based on a boundary but to ignore the landmark as a reference cue for encoding a target location. This hypothesis can explain why an impairment effect resulted from removing a continuous boundary after participants had learned the target locations with compound cues (a boundary and a single landmark), both in previous studies (Doeller \& Burgess, 2008; Mou \& Zhou, 2013) and in Experiment 1 (the ConeArcConeArc condition) of the present study.

One thing to be noted here is that the environmental cues in the present study were mostly organized in a circular shape (as in our previous study, Mou \& Zhou, 2013). This design, to a certain degree, would limit the generalization of the shape-segment hypothesis to cases in which the environmental cues formed irregular shapes. Determining how people would segment an environment when the global shape becomes hard to perceive would demand a similar paradigm using a continuous boundary and a landmark array forming some irregular shapes. Future studies should be conducted to address this issue.

In summary, this study has shown that the global shape formed by testing cues is important for encoding locations: When the global shape information obtained at retrieval was consistent with the global shape (i.e., a circle) formed by the learning cues, recall of the object locations was not impaired; otherwise, location memory was impaired. These findings support the proposal that although the individual reference points provided by a boundary are important for establishing vectors between the local references and targets, the global shape of a boundary nonetheless affects the segmentation process and the subsequent selection of local reference points for encoding locations.
Author note This work was funded by the NSERC, Canada to W.M. None of the data or materials for the experiments reported here are available, and none of the experiments was preregistered.

\section{References}

Barry, C., \& Burgess, N. (2014). Neural mechanisms of self-location. Current Biology, 24, 330-339.

Barry, C., Lever, C., Hayman, R., Hartley, T., Burton, S., O'Keefe, J., \& Burgess, N. (2006). The boundary vector cell model of place cell firing and spatial memory. Reviews in the Neurosciences, 17, 71-97.

Bird, C. M., Capponi, C., King, J. A., Doeller, C. F., \& Burgess, N. (2010). Establishing the boundaries: The hippocampal contribution to imagining scenes. Journal of Neuroscience, 30, 1168811695.

Buckley, M. G., Smith, A. D., \& Haselgrove, M. (2015). Learned predictiveness training modulates biases towards using boundary or landmark cues during navigation. Quarterly Journal of Experimental Psychology, 68, 1183-1202.

Buckley, M. G., Smith, A. D., \& Haselgrove, M. (2019). Thinking outside of the box. II: Disrupting the cognitive map. Cognitive Psychology, 108, 22-41.

Bullens, J., Nardini, M., Doeller, C. F., Braddick, O., Postma, A., \& Burgess, N. (2010). The role of landmarks and boundaries in the development of spatial memory. Developmental Science, 13, 170 180.

Burgess, N. (2008). Spatial cognition and the brain. Annals of the New York Academy of Sciences, 1124, 77-97.

Cheng, K. (1986). A purely geometric module in the rat's spatial representation. Cognition, 23, 149-178.

Cheng, K. (1988). Some psychophysics of the pigeon's use of landmarks. Journal of Comparative Physiology A, 162, 815-826.

Cheng, K. (1989). The vector sum model of pigeon landmark use. Journal of Experimental Psychology: Animal Behavior Processes, $15,366-375$.

Cheng, K., Huttenlocher, J., \& Newcombe, N. S. (2013). 25 years of research on the use of geometry in spatial reorientation: A current theoretical perspective. Psychonomic Bulletin \& Review, 20, 10331054.

Cheng, K., \& Newcombe, N. S. (2005). Is there a geometric module for spatial reorientation? Squaring theory and evidence. Psychonomic Bulletin \& Review, 12, 1-23.

Doeller, C. F., \& Burgess, N. (2008). Distinct error-correcting and incidental learning of location relative to landmarks and boundaries. Proceedings of the National Academy of Sciences, 105, 5909-5914.

Doeller, C. F., King, J. A., \& Burgess, N. (2008). Parallel striatal and hippocampal systems for landmarks and boundaries in spatial memory. Proceedings of the National Academy of Sciences, 105, 59155920.

Foo, P., Warren, W. H., Duchon, A., \& Tarr, M. J. (2005). Do humans integrate routes into a cognitive map? Map- versus landmark-based navigation of novel shortcuts. Journal of Experimental Psychology: Learning, Memory, and Cognition, 31, 195-215.

Gallistel, C.R. (1990). The organization of learning. Cambridge: MIT Press, Bradford Books.

Hermer, L., \& Spelke, E. S. (1994). A geometric process for spatial reorientation in young children. Nature, 370, 57-59. 
Kamil, A.C., \& Cheng, K. (2001). Way-finding and landmarks: The multiple-bearings hypothesis. Journal of Experimental Biology, 204, 103-113.

Keinath, A. T., Julian, J. B., Epstein, R. A., \& Muzzio, I. A. (2017). Environmental geometry aligns the hippocampal map during spatial reorientation. Current Biology, 27, 309-317. https://doi.org/10. 1016/j.cub.2016.11.046

Kelly, D. M., Spetch, M. L., \& Heth, C. D. (1998). Pigeons' (Columba livia) encoding of geometric and featural properties of a spatial environment. Journal of Comparative Psychology, $112,259-269$.

Kosaki, Y., Austen, J. M., \& McGregor, A. (2013). Overshadowing of geometry learning by discrete landmarks in the water maze: Effects of relative salience and relative validity of competing cues. Journal of Experimental Psychology: Animal Behavior Processes, 39, 126139.

Learmonth, A., Nadel, L., \& Newcombe, N. S. (2002). Children's use of landmarks: Implications for modularity theory. Psychological Science, 13, 337-341.

Lee, S. A., Sovrano, V. A., \& Spelke, E. S. (2012). Navigation as a source of geometric knowledge: Young children's use of length, angle, distance, and direction in a reorientation task. Cognition, 123, 144-161.

Lee, S. A., \& Spelke, E. S. (2010). A modular geometric mechanism for reorientation in children. Cognitive Psychology, 61, 152-176.

Lew, A. R. (2011). Looking beyond the boundaries: Time to put landmarks back on the cognitive map? Psychological Bulletin, 137, 484-507.

Miller, N. Y., \& Shettleworth, S. J. (2007). Learning about environmental geometry: An associative model. Journal of Experimental Psychology: Animal Behavior Processes, 33, 191-212.

Mou, W., \& Zhou, R. (2013). Defining a boundary in goal localization: Infinite number of points or extended surfaces. Journal of
Experimental Psychology: Learning, Memory, and Cognition, 39, $1115-1127$.

Newcombe, N. S., \& Ratliff, K. R. (2007). Explaining the development of spatial reorientation: Modularity-plus-language versus the emergence of adaptive combination. In J. Plumert \& J. Spencer (Eds.), The emerging spatial mind (pp. 53-76). New York: Oxford University Press.

O'Keefe, J., \& Burgess, N. (1996). Geometric determinants of the place fields of hippocampal neurons. Nature, 381, 425-428.

Pearce, J. M., Graham, M., Good, M. A., Jones, P. M., \& McGregor, A. (2006). Potentiation, overshadowing, and blocking of spatial learning based on the shape of the environment. Journal of Experimental Psychology: Animal Behavior Processes, 32, 201-214.

Ratliff, K. R., \& Newcombe, N. S. (2008). Reorienting when cues conflict: Evidence for an adaptive-combination view. Psychological Science, 19, 1301-1307.

Sovrano, V. A., Bisazza, A., \& Vallortigara, G. (2002). Modularity and spatial reorientation in a simple mind: Encoding of geometric and nongeometric properties of a spatial environment by fish. Cognition, 85, B51-B59.

Vallortigara, G., Zanforlin, M., \& Pasti, G. (1990). Geometric modules in animals' spatial representations: A test with chicks (Gallus gallus domesticus). Journal of Comparative Psychology, 104, 248-254.

Yoder, R. M., Clark, B. J., \& Taube, J. S. (2011). Origins of landmark encoding in the brain. Trends in Neurosciences, 34, 561-571.

Publisher's note Springer Nature remains neutral with regard to jurisdictional claims in published maps and institutional affiliations. 